Finding Grasping Configuration of a Dexterous Hand and an I ndustrial Robot

J an Rossell, Xavier Sierra, Leopold Palomo and Raúl Suárez

IOC-DT-P-2004-14

Octubre 2004 


\title{
Finding Grasping Configurations of a Dexterous Hand and an Industrial Robot
}

\author{
Jan Rosell, Xavier Sierra, Leopold Palomo and Raúl Suárez \\ Institut d'Organització i Control de Sistemes Industrials (IOC-UPC) \\ Barcelona, Spain. Email: jan.rosell@upc.es
}

\begin{abstract}
Given an industrial robot equipped with a dexterous hand and an object to be grasped with four grasping points determined on its faces, this paper deals with the problem of finding the joint configurations that allow to grasp that object. The proposed solution is based on an iterative optimization method that consecutively moves the joint that best contributes to reduce the distance of the fingertips to the desired locations. The method is particularized for a Stäubli RX90 robot and the dexterous hand MA-I with four fingers developed at the IOC's Robotics Lab.
\end{abstract}

\section{INTRODUCTION}

Dexterous hands are incorporated to robots in order to make them more flexible and widen the type of tasks they can perform. This fact involves many specific planning and control problems. The first problem to be tackled is the grasp synthesis that determines the best stable and proper grasp for a given object [1], i.e. where should the fingers be placed on the object and in which direction should the forces be exerted in order to grasp the object.

Once the grasping points on the object are known, the inverse kinematics of the hand-arm ensemble must be solved in order to determine the joint positions for the actual configuration of the object. This is a complex problem due to the great number of involved degrees of freedom and the tree structure of the kinematic chain.

In this paper a general method to solve the inverse kinematic problem for a hand-arm ensemble is introduced. The proposal is based on an optimization method that can cope with general robots and hands, although it is particularized for a Stäubli RX90 robot and the dexterous hand MA-I with four fingers developed at the IOC's Robotics Lab [2].

After this introduction, the paper is structured as follows. Section II describes the problem by presenting the kinematic model, the problem statement and the proposed solution based on an iterative optimization method. Section III presents the objective functions to be minimized, and Section IV describes the proposed inverse kinematics algorithm. The proposed method is validated with the experiments reported in Section V. Finally, Section VI presents the conclusions of the work.

\section{PROBlem DESCRIPTION}

\section{A. Kinematic model}

A robot arm equipped with a mechanical hand form a kinematic tree structure. Let $A$ and $F$ be the number of degrees of freedom of the arm and of each finger, respectively, and $N$ be $N=A+F$. Let $K$ be the number of fingers of the hand. The kinematic tree structure is considered as $K$ kinematic chains that share the first $A$ links. Then the links are labelled as $j_{k}$ with $j=1, \ldots, N$ and $k=1, \ldots, K$. When the meaning is clear, the subindex will be omitted for the arm links, since $j_{m}=j_{n}$ $\forall m, n \in\{1, \ldots, K\}$ and $\forall j \in\{1, \ldots, A\}$.

Using this nomenclature, let us define the following reference frames (Figure 1):

- $\mathcal{F}_{W}$ : world reference frame.

- $\mathcal{F}_{0}$ : reference frame attached to the base of the robot.

- $\mathcal{F}_{j_{k}}$ : reference frame attached to link $j_{k}$. The frames attached to the fingertips are called $\mathcal{F}_{N_{k}}$, with $k=1, \ldots, K$.

- $\mathcal{F}_{N_{k}^{*}}$ : desired position of $\mathcal{F}_{N_{k}}$.

The reference frames $\mathcal{F}_{0}, \mathcal{F}_{N_{k}^{*}}$ and $\mathcal{F}_{N_{k}}$ are described, with respect to frame $\mathcal{F}_{W}$, by homogeneous transformations $T_{W}^{0}, T_{W}^{N_{k}^{*}}$ and $T_{W}^{N_{k}}$, respectively. Each reference frame $\mathcal{F}_{j_{k}}$ is described with respect to the previous link reference frame, $\mathcal{F}_{(j-1)_{k}}$, by means of a homogeneous transformation, $T_{(j-1)_{k}}^{j_{k}}$. Using the Denavit-Hartenberg parameters $\alpha_{j_{k}}, \theta_{j_{k}}, a_{j_{k}}$ and $d_{j_{k}}, T_{(j-1)_{k}}^{j_{k}}$ is given by [3]:

$$
T_{(j-1)_{k}}^{j_{k}}=\left[\begin{array}{cccc}
\cos \theta_{j_{k}}-\cos \alpha_{j_{k}} \sin \theta_{j_{k}} & \sin \alpha_{j_{k}} \sin \theta_{j_{k}} & a_{j_{k}} \cos \theta_{j_{k}} \\
\sin \theta_{j_{k}} & \cos \alpha_{j_{k}} \cos \theta_{j_{k}} & -\sin \alpha_{j_{k}} \cos \theta_{j_{k}} & a_{j_{k}} \sin \theta_{j_{k}} \\
0 & \sin \theta_{j_{k}} & \cos \alpha_{j_{k}} & d_{j_{k}} \\
0 & 0 & 0 & 1
\end{array}\right]
$$

This work was partially supported by the CICYT projects DPI2004-03104 and DPI2002-03540 


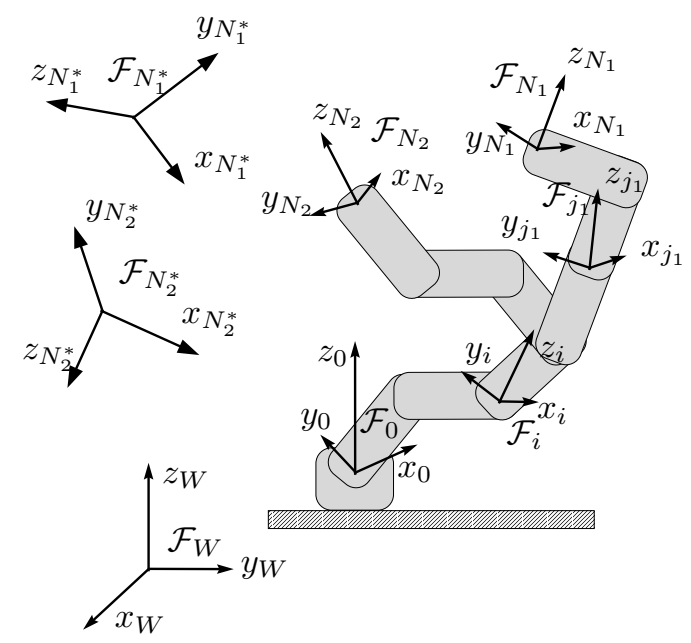

Fig. 1. Definition of reference frames.

Finger 1

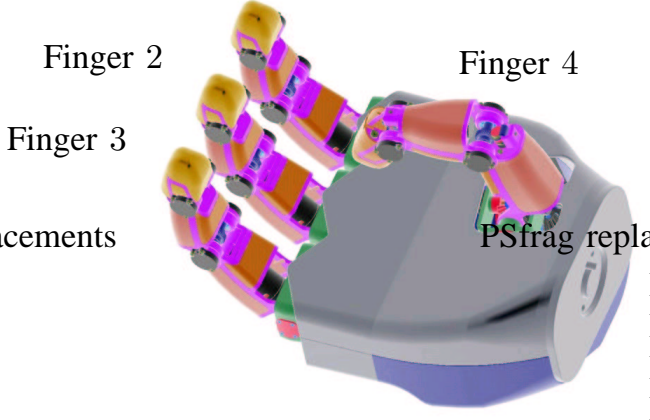

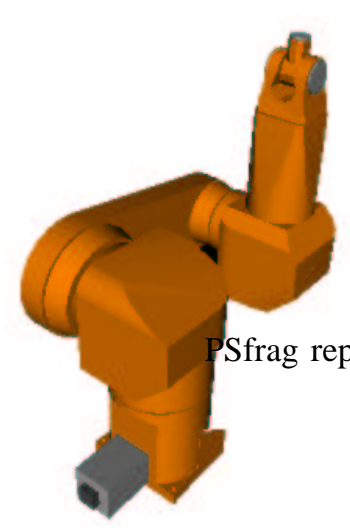

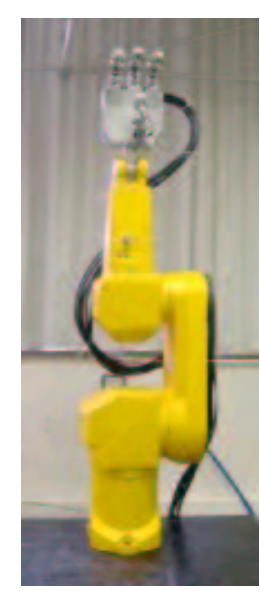

Fig. 2. CAD models of the dexterous hand MA-I and the Stäubli RX90 robot and the physical hand-arm ensemble.

\section{B. Particular hand-arm ensemble}

Figure 2 show the CAD models of the hand MA-I and the robot Stäubli RX90 as well as the real hand-arm ensemble.

The dexterous hand MA-I developed at the IOC's Robotics Lab has four fingers with four degrees of freedom each one. Three additional virtual joints are considered at the fingertips in order to take into consideration that (Figure 3):

- Any point of the fingertip can be used to contact with the selected grasping point on the object. Assuming a spherical fingertip, the contact fingertip point can be determined by two virtual joints, $\theta_{11_{k}}$ and $\theta_{12_{k}}$, and a virtual link of length the radius of the sphere. The range of these virtual joints determines the valid area of the fingertip sphere.

- Only the direction normal to the object surface at the grasping contact point is defined as a requirement for the grasping and, therefore, the orientation of the finger around this normal is free. This is modelled by another virtual joint, $\theta_{13_{k}}$, with a range of $2 \pi$.

The DH parameters of the hand and the arm, including the three virtual joints of the fingertips, are shown in the Appendix.

\section{Problem statement and proposed solution}

The problem to be solved is "which are the proper positions of the arm and hand joints in order to perform a given grasp?", i.e. find the set of joint values that locate the fingertip reference frames $\mathcal{F}_{N_{k}}$ at given desired locations $\mathcal{F}_{N_{k}^{*}}$ with $k=1, \ldots, K$.

The proposed solution to this inverse kinematics problem is based on an iterative optimization method. The objective function to be optimized (minimized) is the distance from the current to the desired fingertip locations. The problem is decoupled by analyzing the effect of the individual motion of each joint in this objective function, i.e. the problem is partitioned into several one-degree of freedom optimization subproblems. At each step of the iterative process, the joint value that individually minimize this objective function is computed and used to update the kinematic structure. 

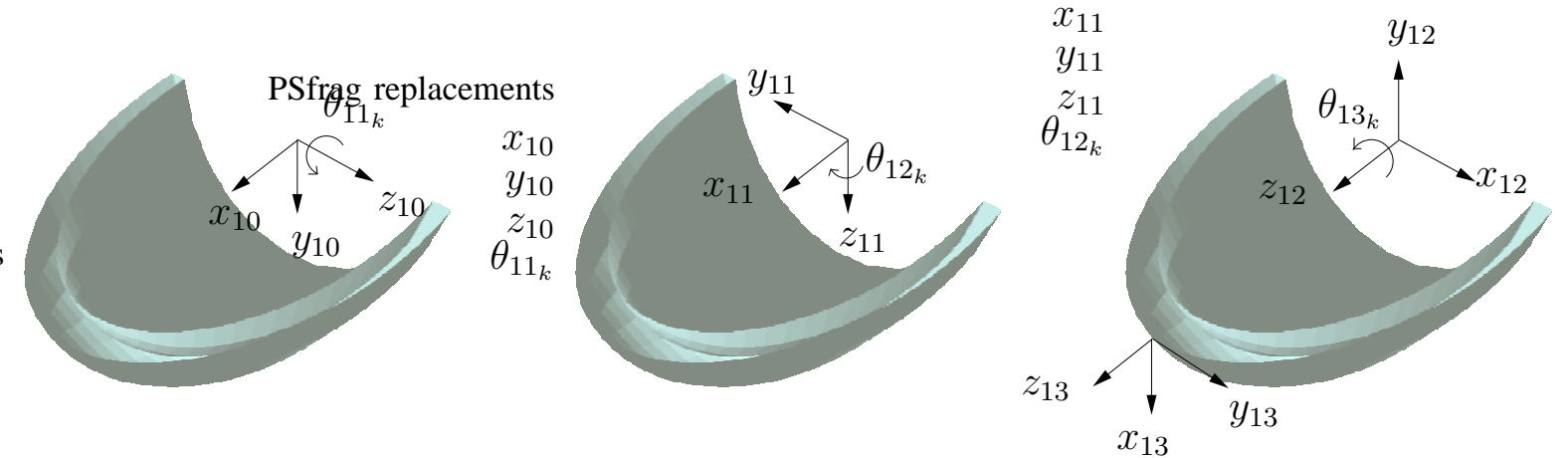

Fig. 3. Fingertip with the virtual revolute joints $\theta_{11_{k}}, \theta_{12_{k}}$ and $\theta_{13_{k}}$ defined around the $z$-axis of $\mathcal{F}_{10_{k}}, \mathcal{F}_{11_{k}}, \mathcal{F}_{12_{k}}$, respectively.

The proposed solution is inspired by the Distributed Optimization Method introduced by Regnier et al. [4] to solve the inverse kinematics of all serial manipulators, and also used to solve the problem of the kinematic synthesis of manipulators [5]. A similar approach, but using a different distance metrics, was presented in [6] to solve the inverse kinematics problem for redundant manipulators. The solution proposed in this paper uses a different distance metrics and extends the approach to kinematic-tree structures.

\section{OBJECTIVE FunCTIONS}

\section{A. Distance metrics}

The representation of the distance between two reference frames involves the parameterized mix of translational and rotational components. No bi-invariant metrics exist in SE(3), the Euclidean group of rigid-body motions, although left- or right-invariant distance metrics can be proposed [7] (i.e. distances invariant with respect to the choice of the inertial frame or to the choice of the rigid body frame, respectively). The use of these metrics can be computationally expensive and therefore simpler metrics are usually proposed in iterative procedures (e.g. [4], [6]). These simpler metrics may not have such invariant features nor a well-defined mix of translational and rotational components. These factors may influence the behavior of metric-based complex algorithms in an unclear and, therefore, undesirable way. As a consequence, the left-invariant metric proposed in [7] is used in this paper.

Let $X_{1}$ and $X_{2}$ be two homogeneous transformations defining two reference frames. Then the distance between them is determined by:

$$
\operatorname{dist}\left(X_{1}, X_{2}\right)=\sqrt{\phi^{2}+\frac{1}{L^{2}} \Delta^{2}}
$$

where $\phi$ is the angle, around a given axis, that $X_{1}$ must rotate in order to obtain the same orientation as $X_{2}, \Delta$ is the euclidian distance between the origins, and $L$ is a parameter that weights the translational and rotational components.

The translational distance $\Delta$ and the rotational distance $\phi$ are computed in the following subsections considering the kinematic chain formed by the robot and one finger assuming $\mathcal{F}_{W}=\mathcal{F}_{0}$. In this case $\mathcal{F}_{N_{k}^{*}}$ and $\mathcal{F}_{N_{k}}$ are described, respectively, by $T_{0}^{N_{k}^{*}}$ and $T_{0}^{N_{k}}$. Therefore:

$$
\operatorname{dist}\left(X_{1}, X_{2}\right)=\operatorname{dist}\left(T_{0}^{N_{k}}, T_{0}^{N_{k}^{*}}\right)
$$

Since this is a left invariant distance, this equation can be reformulated as (Figure 4):

$$
\operatorname{dist}\left(X_{1}, X_{2}\right)=\operatorname{dist}\left(T_{(j-1)_{k}}^{j_{k}} \cdot T_{j_{k}}^{N_{k}},\left(T_{0}^{(j-1)_{k}}\right)^{-1} \cdot T_{0}^{N_{k}^{*}}\right)
$$

This equation is written considering $\theta_{j_{k}}$ as the unique variable (i.e. considering fixed all other joint values). This allows to find the value of $\theta_{j_{k}}$ that minimizes the distance. Let the translational and rotational components be called $\Delta_{j_{k}}$ and $\phi_{j_{k}}$, respectively. Their expressions are the followings: 
1) Translational distance: Let the homogeneous transformations $T_{j_{k}}^{N_{k}}$ and $\left[\left(T_{0}^{j_{k}-1}\right)^{-1} \cdot T_{0}^{N_{k}^{*}}\right]$ be expressed as:

$$
\begin{aligned}
T_{j_{k}}^{N_{k}} & =\left[\begin{array}{cccc}
t_{00} & t_{01} & t_{02} & t_{03} \\
t_{10} & t_{11} & t_{12} & t_{13} \\
t_{20} & t_{21} & t_{22} & t_{23} \\
0 & 0 & 0 & 1
\end{array}\right] \\
{\left[\left(T_{0}^{(j-1)_{k}}\right)^{-1} \cdot T_{0}^{N_{k}^{*}}\right]=} & {\left[\begin{array}{cccc}
h_{00} & h_{01} & h_{02} & h_{03} \\
h_{10} & h_{11} & h_{12} & h_{13} \\
h_{20} & h_{21} & h_{22} & h_{23} \\
0 & 0 & 0 & 1
\end{array}\right] }
\end{aligned}
$$

Then, being $T_{(j-1)_{k}}^{j_{k}}$ defined by equation (1), the square of the translational distance $\Delta_{j_{k}}$ is:

$$
\begin{aligned}
\Delta_{j_{k}}^{2}= & P_{j_{k}} \cos \left(\theta_{j_{k}}\right)+Q_{j_{k}} \sin \left(\theta_{j_{k}}\right)+R_{j_{k}} \\
P_{j_{k}}= & 2\left(-t_{03} h_{03}-\cos \alpha_{j_{k}} t_{13} h_{13}+\sin \alpha_{j_{k}} t_{23} h_{13}-a_{j_{k}} h_{03}\right) \\
Q_{j_{k}}= & 2\left(-t_{03} h_{13}-\cos \alpha_{j_{k}} t_{13} h_{03}-\sin \alpha_{j_{k}} t_{23} h_{03}-a_{j_{k}} h_{13}\right) \\
R_{j_{k}}=2 & \cos \alpha_{j_{k}}\left(t_{23} d_{j_{k}}-t_{23} h_{23}\right)+2 \sin \alpha_{j_{k}}\left(t_{13} d_{j_{k}}-t_{13} h_{23}\right)+ \\
& h_{03}^{2}+t_{13}^{2}+t_{23}^{2}+t_{03}^{2}+a_{j_{k}}^{2}+h_{13}^{2}+ \\
& d_{j_{k}}^{2}-2 d_{j_{k}} h_{23}+h_{23}^{2}+2 t_{03} a_{j_{k}}
\end{aligned}
$$

2) Rotational distance: If $\Phi_{1}$ and $\Phi_{2}$ are the rotation matrices associated to $\left[\left(T_{0}^{(j-1)_{k}}\right)^{-1} \cdot T_{0}^{N_{k}^{*}}\right]$ and $\left[T_{(j-1)_{k}}^{j_{k}} \cdot T_{j_{k}}^{N_{k}}\right]$, respectively, then [7]:

$$
\phi_{j_{k}}^{2}=\arccos ^{2}\left(\frac{\operatorname{tr}\left(\Phi_{1}^{-1} \Phi_{2}\right)-1}{2}\right)
$$

where $\operatorname{tr}(\cdot)$ means the trace of a matrix.

This expression can be approximated by the following one:

$$
\phi_{j_{k}}^{2}=\frac{\pi^{2}}{2}\left(1-\frac{\operatorname{tr}\left(\Phi_{1}^{-1} \Phi_{2}\right)-1}{2}\right)
$$

If $\Phi_{1}^{-1}$ is expressed as:

$$
\Phi_{1}^{-1}=\left[\begin{array}{ccc}
a_{00} & a_{01} & a_{02} \\
a_{10} & a_{11} & a_{12} \\
a_{20} & a_{21} & a_{22}
\end{array}\right]
$$

Then, the square of the rotational distance $\phi_{j_{k}}$ is:

$$
\begin{aligned}
\phi_{j_{k}}^{2}= & \frac{\pi^{2}}{4}\left(3-A_{j_{k}} \cos \left(\theta_{j_{k}}\right)-B_{j_{k}} \sin \left(\theta_{j_{k}}\right)-C_{j_{k}}\right) \\
A_{j_{k}}= & a_{00} t_{00}+a_{10} t_{01}+a_{20} t_{02}+ \\
& \cos \alpha_{j_{k}}\left(a_{01} t_{10}+a_{11} t_{11}+a_{21} t_{12}\right)- \\
& -\sin \alpha_{j_{k}}\left(a_{01} t_{20}+a_{11} t_{21}+a_{21} t_{22}\right) \\
B_{j_{k}}= & a_{01} t_{00}+a_{11} t_{01}+a_{21} t_{02}- \\
& \cos \alpha_{j_{k}}\left(a_{00} t_{10}+a_{10} t_{11}+a_{20} t_{12}\right)+ \\
& +\sin \alpha_{j_{k}}\left(a_{00} t_{20}+a_{10} t_{21}+a_{20} t_{22}\right) \\
C_{j_{k}}= & \cos \alpha_{j_{k}}\left(a_{02} t_{20}+a_{12} t_{21}+a_{22} t_{22}\right)+ \\
& \sin \alpha_{j_{k}}\left(a_{02} t_{10}+a_{12} t_{11}+a_{22} t_{12}\right)
\end{aligned}
$$

\section{B. Objective functions for finger joints}

The inverse kinematics problem can be reformulated as a set of one degree of freedom optimization subproblems, with the following objective functions to be minimized:

$$
F_{j_{k}}=\operatorname{dist}\left(T_{(j-1)_{k}}^{j_{k}} \cdot T_{j_{k}}^{N_{k}},\left(T_{0}^{(j-1)_{k}}\right)^{-1} \cdot T_{0}^{N_{k}^{*}}\right)
$$




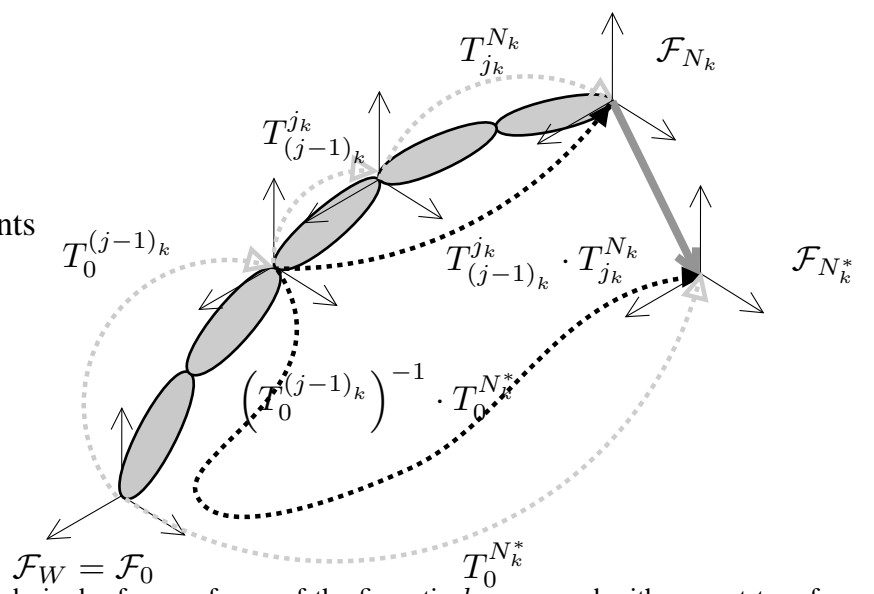

Fig. 4. Distance from the current to the desired reference frame of the fingertip $k$, measured with respect to reference frame of link $j_{k}$ (using $T_{W}^{0}=I$ ).

i.e. the distance from the current to the desired location of the fingertip reference frame is rewritten as a function of each joint $\theta_{j_{k}}$ with $j=(A+1), \ldots,(A+F), k=1, \ldots, K$. Taking into account equations (2),(7) and (11), the resulting objective function is:

$$
\begin{aligned}
F_{j_{k}}= & \frac{\pi^{2}}{4}\left(3-A_{j_{k}} \cos \theta_{j_{k}}-B_{j_{k}} \sin \theta_{j_{k}}-C_{j_{k}}\right)+ \\
& \frac{1}{L^{2}}\left(P_{j_{k}} \cos \theta_{j_{k}}+Q_{j_{k}} \sin \theta_{j_{k}}+R_{j_{k}}\right)
\end{aligned}
$$

This expression can be rewritten as:

$$
\begin{aligned}
F_{j_{k}} & =\alpha_{j_{k}} \cos \theta_{j_{k}}+\beta_{j_{k}} \sin \theta_{j_{k}}+\gamma_{j_{k}} \\
\alpha_{j_{k}} & =\frac{1}{L^{2}} P_{j_{k}}-\frac{\pi^{2}}{4} A_{j_{k}} \\
\beta_{j_{k}} & =\frac{1}{L^{2}} Q_{j_{k}}-\frac{\pi^{2}}{4} B_{j_{k}} \\
\gamma_{j_{k}} & =\frac{\pi^{2}}{4}\left(3-C_{j_{k}}\right)+\frac{1}{L^{2}} R_{j_{k}}
\end{aligned}
$$

\section{Objective functions for arm joints}

The motion of the arm joints affect the position and orientation of the reference frames of all the fingers. Therefore, a different objective function for these joints must be defined in order to take into account this fact.

Let $F_{j}^{k}$ be the objective function that gives the distance from the current to the desired position of the fingertip $k$, measured in the reference frame of the arm link $j$, with $j=1, \ldots, A$.

The function $F_{j}^{k}$ is expressed by equation (14), since a unique kinematic serial chain is considered. Then, in order to consider all the kinematic chains, the objective function $F_{j}$ of the arm joint $\theta_{j}$ is computed as the sum of the functions $F_{j}^{k}$, with $k \in 1 \ldots K$ :

$$
F_{j}=\sum_{k=1}^{K} F_{j}^{k}=\sum_{k=1}^{K} \alpha_{j_{k}} \cos \theta_{j_{k}}+\beta_{j_{k}} \sin \theta_{j_{k}}+\gamma_{j_{k}}
$$

Using the DH notation, the $z$ axis of the reference frame $\mathcal{F}_{j}$ is set coincident with the axis of the joint $\theta_{j+1}$. This poses a problem for the last link of the arm, since with this convention $K$ reference frames are defined at link $A$ and therefore $K$ measures of joint $\theta_{A}$ are obtained, although they differ only by a constant offset value [8]. In the proposed approach, the correspondence between these values is arbitrarily set with respect to finger 1 :

$$
\theta_{A_{k}}=\theta_{A_{1}}+\delta_{A_{k}} \quad k=1 \ldots K
$$

with $\delta_{A_{1}}=0$ and $\delta_{A_{k}}$ dependant on the geometry. For the hand MA-I these offset values are $\delta_{A_{2}}=\delta_{A_{3}}=0$ and $\delta_{A_{4}}=33.7^{\circ}$.

In order to take into account this fact, equation (15) has to be modified for joint $\theta_{A}$ as follows:

$$
F_{A}=\sum_{k=1}^{K} \alpha_{A_{k}} \cos \left(\theta_{A_{1}}+\delta_{A_{k}}\right)+\beta_{A_{k}} \sin \left(\theta_{A_{1}}+\delta_{A_{k}}\right)+\gamma_{A_{k}}
$$




\section{Minimization of the objective function}

The objective functions presented in the previous sections measure the distance from the current to the desired location of the fingertips, as a function of each single joint value. Then, these objective functions can be minimized to obtain the optimum joint values, i.e. the values of the joints that move the fingertips close to their desired location.

The joint value that minimizes $F_{j_{k}}$ is obtained from $\frac{\partial F_{j_{k}}}{\partial \theta_{j_{k}}}=0$ using:

- equation (15) for links $j=1, \ldots,(A-1)$

- equation (17) for link $j=A$

- equation (14) for links $j_{k}$ with $k=1, \ldots, K$ and $j=(A+1), \ldots,(A+F)$.

The value $\theta_{j_{k}}$ obtained is:

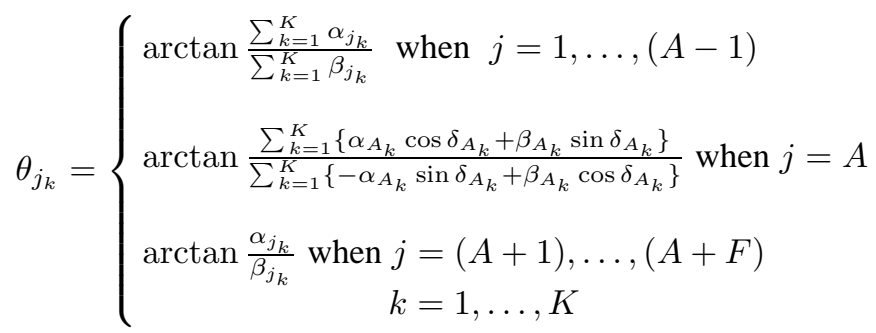

The value of $\theta_{j_{k}}$ is checked to be a minimum by verifying that the sign of the second derivative is positive. If this is not the case, the minimum occurs at $\left(\theta_{j_{k}}+\pi\right)$.

When $\theta_{j_{k}}$ is outside the range $\left[\theta_{j_{k}}^{\max }, \theta_{j_{k}}^{\min }\right]$ of possible joint values, it is set to the limit value:

$$
\begin{aligned}
& \text { if } \theta_{j_{k}}<\theta_{j_{k}}^{\text {min }} \text { then } \theta_{j_{k}}=\theta_{j_{k}}^{\text {min }} \\
& \text { if } \theta_{j_{k}}>\theta_{j_{k}}^{\text {max }} \text { then } \theta_{j_{k}}=\theta_{j_{k}}^{\text {max }}
\end{aligned}
$$

\section{Optimization METHOD}

The inverse kinematics of the hand-arm ensemble is solved with an optimization method based on a procedure that iteratively computes the objective functions and moves the joint that best approaches the hand to the desired configuration.

The success of the proposed method depends on the initial joint values, due to the nature of iterative optimization algorithms. When the improvement of the iterative procedure is not good enough (measured as a relative decrement of the objective function), a retrial is performed restarting the procedure from a new initial configuration. A deterministic sampling sequence is used to uniformly generate initial configurations over the configuration space.

\section{A. Hand-arm inverse kinematics algorithm}

The algorithm to compute the hand-arm inverse kinematics, shown below, uses the following functions:

OptimizeArm: This function uses equation (18) for $j=1, \ldots, A$ to find the value of the arm joint that minimizes the mean distance from $\mathcal{F}_{N_{k}}$ to $\mathcal{F}_{N_{k}^{*}}$ with $k=1, \ldots, K$. The function returns this optimum joint value.

OptimizeFinger: For a given finger $k$, this function uses equation (18) for $j=(A+1), \ldots,(A+F)$ to find the value of the finger joint that minimizes the distance from $\mathcal{F}_{N_{k}}$ to $\mathcal{F}_{N_{k}^{*}}$. The function returns:

- this optimum joint value.

- the value of the objective function, $\mathbf{F}_{k}$

MoveJoint: This function moves a specified joint, $j_{k}$, to the given value, $\theta_{j_{k}}$.

StartConfiguration: This function generates the initial values of the arm joints using a deterministic sampling sequence. The initial finger joints values are set to the middle value of the corresponding ranges. The function returns a vector $\Theta^{i n i}$ with those values. 


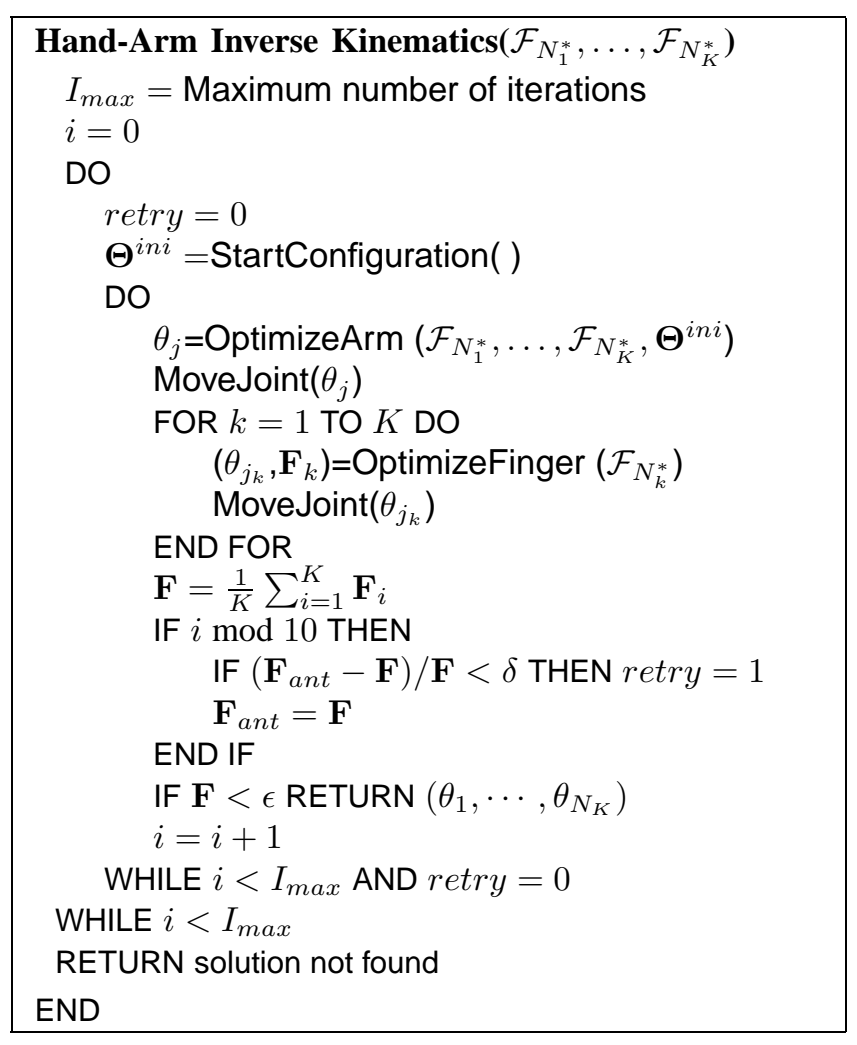

\section{B. Initial configurations}

The initial configurations of the arm joints are determined by sampling the corresponding configuration space. This problem is the same as the one encountered in sampling-based motion planners. Usually these planners randomly sample the configuration space (often with an heuristic bias towards the regions where it is most difficult to find a path). However, other sampling-based motion planners rely on deterministic sampling sequences. Deterministic sampling provide a good uniform and incremental coverage of the space, and can outperform random sampling in nearly all motion planning problems [9]. The determination of the initial configurations of the proposed optimization method has been done using both random and deterministic sampling [10]. The best results where obtained using deterministic sampling.

\section{EXPERIMENTS}

The inverse kinematics algorithm has been incorporated to the Qilex robotics simulator developed at the IOC's Robotic Lab (http://qilex.berlios.de/, Figure 5), and has been statistically tested. The validation consisted of:

- Generating a set of grasping configurations by randomly setting the values of all the joints of the hand-arm ensemble and then computing the direct kinematics.

- Applying the inverse kinematics algorithm to the test set.

The test set is composed of 1,000 grasping configurations. The algorithm has been able to find the solution of the inverse kinematics in the $100 \%$ of the cases, using $I_{\max }=25,000, \epsilon=0.00001$ and $\delta=0.01$. The value that weighs the translational distance is fixed at $L=80$. The mean number of iterations was 3,997 and the mean number of retrials was 18 . The histograms of the corresponding results for the 1,000 test configurations are shown in Figures 6 and 7.

\section{CONCLusions}

The use of dexterous hands in industrial robots pose several difficult problems, one of them being the determination of the arm and finger joints in order to grasp a given object (once the grasping points on its surface and the direction of the forces to be exerted are known). A general iterative optimization method has been proposed to solve the inverse kinematics problem of hand-arm ensembles. The method consecutively finds the joint motions that best contributes to reach the goal. The objective functions to be minimized are the distances from the fingertips to the grasping points. Distance metrics has been carefully handled in order to properly consider orientations. The solution has been particularized for a Stäubli RX90 robot and the dexterous hand MA-I with four fingers developed at the IOC's Robotics Lab. The approach has been validated through exhaustive experiments on a simulator. 


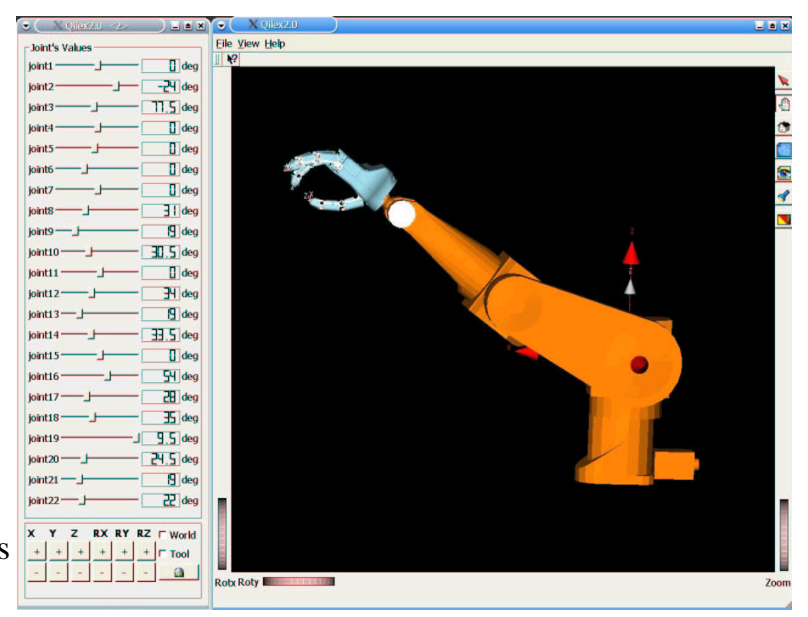

Fig. 5. Cell simulator with the RX90 robot and the MA-I hand.

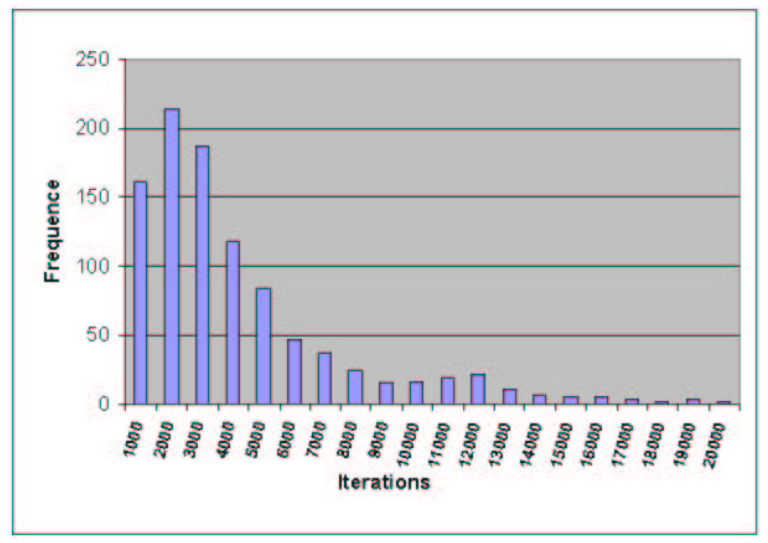

Fig. 6. Histogram of number of iterations.

\section{APPENDIX}

The Denavit-Hartenberg parameters of the fingers of MA-I and the robot Stäubli RX90 are detailed in the the following tables (angles expressed in degrees and distances in millimeters). Note that the parameters of the last joint of the robot, $\theta_{6}$, are expressed in each finger table with different values due to the feature of the DH notation commented in Section III-C.

RX90 Stäubli robot (joints $j_{k} j=1, \ldots, 5 ; \forall k$ )

\begin{tabular}{|c|c|c|c|c|c|}
\hline$j_{k}$ & 1 & 2 & 3 & 4 & 5 \\
\hline$\alpha_{j_{k}}$ & -90 & 0 & 90 & -90 & 90 \\
\hline$a_{j_{k}}$ & 0 & 450 & 0 & 0 & 0 \\
\hline$d_{j_{k}}$ & 0 & 0 & 0 & 450 & 0 \\
\hline$\theta_{j_{k}}^{\text {min }}$ & -160 & -227.5 & -52.5 & -270 & -105 \\
\hline$\theta_{j_{k}}^{\text {max }}$ & 160 & 47.5 & 232.5 & 270 & 120 \\
\hline
\end{tabular}




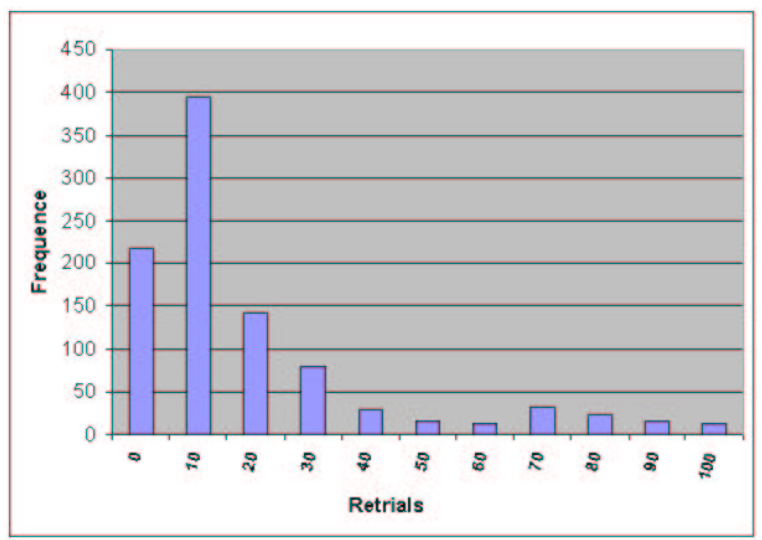

Fig. 7. Histogram of number of retrials.

Finger 1 (joints $j_{k}, j=6, \ldots, 13 ; k=1$ )

\begin{tabular}{|c|c|c|c|c|c|c|c|c|}
\hline$j_{k}$ & 6 & 7 & 8 & 9 & 10 & 11 & 12 & 13 \\
\hline$\alpha_{j_{k}}$ & 90 & 90 & 0 & 0 & 0 & -90 & -90 & 0 \\
\hline$a_{j_{k}}$ & 67 & 0 & 76 & 56 & 40 & 0 & 0 & 0 \\
\hline$d_{j_{k}}$ & 276 & 11 & 0 & 0 & 0 & 0 & 0 & 15 \\
\hline$\theta_{j_{k}}^{\text {min }}$ & -180 & 80 & 0 & 0 & 0 & 0 & -180 & -270 \\
\hline$\theta_{j_{k}}^{\text {max }}$ & 360 & 100 & 90 & 90 & 90 & 90 & 0 & 90 \\
\hline
\end{tabular}

Finger 2 (joints $j_{k}, j=6, \ldots, 13 ; k=2$ )

\begin{tabular}{|c|c|c|c|c|c|c|c|c|}
\hline$j_{k}$ & 6 & 7 & 8 & 9 & 10 & 11 & 12 & 13 \\
\hline$\alpha_{j_{k}}$ & 90 & 90 & 0 & 0 & 0 & -90 & -90 & 0 \\
\hline$a_{j_{k}}$ & 0 & 0 & 0 & 56 & 40 & 0 & 0 & 0 \\
\hline$d_{j_{k}}$ & 276 & 11 & 0 & 0 & 0 & 0 & 0 & 15 \\
\hline$\theta_{j_{k} \text { min }}^{\text {min }}$ & -180 & 80 & 0 & 0 & 0 & 0 & -180 & -270 \\
\hline$\theta_{j_{k} \text { max }}^{\text {max }}$ & 360 & 100 & 90 & 90 & 90 & 90 & 0 & 90 \\
\hline
\end{tabular}

Finger 3 (joints $j_{k}, j=6, \ldots, 13 ; k=3$ )

\begin{tabular}{|c|c|c|c|c|c|c|c|c|}
\hline$j_{k}$ & 6 & 7 & 8 & 9 & 10 & 11 & 12 & 13 \\
\hline$\alpha_{j_{k}}$ & 90 & 90 & 0 & 0 & 0 & -90 & -90 & 0 \\
\hline$a_{j_{k}}$ & -67 & 0 & 76 & 56 & 40 & 0 & 0 & 0 \\
\hline$d_{j_{k}}$ & 276 & 11 & 0 & 0 & 0 & 0 & 0 & 15 \\
\hline$\theta_{j_{k}}^{\text {min }}$ & -180 & 80 & 0 & 0 & 0 & 0 & -180 & -270 \\
\hline$\theta_{j_{k}}^{\text {max }}$ & 360 & 100 & 90 & 90 & 90 & 90 & 0 & 90 \\
\hline
\end{tabular}

Finger 4 (joints $j_{k}, j=6, \ldots, 13 ; k=4$ )

\begin{tabular}{|c|c|c|c|c|c|c|c|c|}
\hline$j_{k}$ & 6 & 7 & 8 & 9 & 10 & 11 & 12 & 13 \\
\hline$\alpha_{j_{k}}$ & 0 & 90 & 0 & 0 & 0 & -90 & -90 & 0 \\
\hline$a_{j_{k}}$ & 72 & 0 & 76 & 66 & 45 & 0 & 0 & 0 \\
\hline$d_{j_{k}}$ & 145 & 0 & 0 & 0 & 0 & 0 & 0 & 15 \\
\hline$\theta_{j_{k}}^{\text {min }}$ & -213.7 & -66.3 & 0 & 0 & 0 & 0 & -180 & -270 \\
\hline$\theta_{j_{k}}^{\text {max }}$ & 326.3 & -46.3 & 90 & 90 & 90 & 90 & 0 & 90 \\
\hline
\end{tabular}

\section{REFERENCES}

[1] K. B. Shimoga, "Robot grasp synthesis algorithms: A survey," The Int. Journal of Robotics Research, vol. 15, no. 3, pp. 230-266, 1996.

[2] R. Suárez and P. Grosch, "Dexterous robotic hand ma-i, sofware and hardware architecture," in Int. Conf. Intelligent Manipulation and Grasping, 2004, pp. pp. 91-96.

[3] J. Denavit and R. Hartenberg, "A kinematic notation for lower-pair mechanisms based on matrices," Journal of Applied Mechanics, vol. 22, pp. 215-221, June 1955.

[4] S. Regnier, F. B. Ouenzou, and P. Bidaud, "Distributed optimization method for inverse kinematics of all serial manipulators," Journal of Mechanical and Machine Theory, vol. 32, no. 7, pp. 1-15, October 1997.

[5] F. B. Ouezdou, S. Regnier, and C. Mavroidis, "Kinematic synthesis of manipulators using a distributed optimization method," Journal of Mechanical Design, Transactions of the ASME, vol. 121, no. 4, pp. 492-501, 1999.

[6] J. M. Ahuactzin and K. K. Gupta, "The kinematic roadmap: a motion planning based global approach for inverse kinematics of redundant robots," IEEE Trans. on Robotics and Automation, vol. 15, no. 4, pp. 653 -669, Aug. 1999. 
[7] F. C. Park, "Distance metrics on the rigid-body motions with applications to mechanical design," Journal of Mechanical Design, vol. 117, pp. 48-54, 1995.

[8] W. Khalil and J. F. Kleinfinger, "A new geometric notation for open and closed-loop robots," in Proc. of the IEEE Int. Conf. on Robotics and Automation, 1986, pp. 1174-1179.

[9] S. R. Lindemann and S. M. LaValle, "Incremental low-discrepancy lattice methods for motion planning," in Proc. of the IEEE Int. Conf. on Robotics and Automation, 2004, pp. 2920-2927.

[10] J. Rosell and P. I. niguez, "An adaptive deterministic sequence for sampling-based motion planners," Tech. Report UPC, IOC-DT-P-2004-26, 2004. Submitted to a Journal. 